\title{
QoS Parametric Inspection of Uniform and Assorted Trajectories for MANET Routing Protocols
}

\author{
Nitesh Sehwani' ${ }^{1}$, Sajid Rahman², Anna Harris ${ }^{2}$ \\ ${ }^{1}$ Departmentof Electronics and Telecommunications Engineering, Sardar Patel Institute of Technology, Mumbai, India \\ ${ }^{2}$ Department of Math and Computer Science, University of Arkansas at Pine Bluff, Pine Bluff, AR, USA \\ Email: niteshsehwani@gmail.com, mailsajidtennis@gmail.com, harrisa@uapb.edu
}

How to cite this paper: Sehwani, N., Rahman, S. and Harris, A. (2017) QoS Parametric Inspection of Uniform and Assorted Trajectories for MANET Routing Protocols. Int. J. Communications, Network and System Sciences, 10, 234-250.

https://doi.org/10.4236/ijcns.2017.1010014

Received: April 30, 2017

Accepted: October 27, 2017

Published: October 30, 2017

Copyright $\odot 2017$ by authors and Scientific Research Publishing Inc. This work is licensed under the Creative Commons Attribution International License (CC BY 4.0).

http://creativecommons.org/licenses/by/4.0/ (c) (i) Open Access

\begin{abstract}
A Mobile Ad Hoc Network (MANET) is a self-governing network of mobile nodes without the inclusion of any wired links. Each node can move in an ad hoc manner and therefore, such a network should consist of routing protocols which can adapt to dynamically changing topologies. Numerous protocols have been proposed for the same. However, the trajectories followed by the individual nodes have not been distinctly dealt with. This paper presents a meticulous study on QoS parameters of proactive (OLSR) and reactive (DSR) protocols of MANETs for uniform as well as dissimilar trajectories of individual nodes in a small network of about 20 nodes. Also an examination of partial node failures for both the above mentioned protocols has been done. The performance metrics utilized in this study are average throughput and average delay. OPNET modeler has been utilized for this study. This assessment shows that for uniform trajectories, OLSR has almost same average delay but a higher average throughput as compared to DSR. Also it is seen that, as compared to uniform trajectories, non-uniform trajectories deliver a much higher average throughput. Node failures only reduce average throughputs whereas average delays remain unchanged.
\end{abstract}

\section{Keywords}

MANET, OLSR, DSR, Routing, Throughput, Delay

\section{Introduction}

MOBILE ad hoc networks (MANETs) consist of moving nodes that use wireless transmission for communication. They can be set up as and when needed due to 
the absence of a centralized setup. Similar to wired networks, data transfers in a MANET have varied characteristics (e.g. category and amount of data exchanged, duration of interplay, etc.) and also different Quality of Service (QoS) [1] requirements (e.g. delay, throughput). Hence, a uniform packet processing is not adequate and a QoS inspection consisting of various parameters is required.

The primary challenge in building a MANET is equipping each device (node) to continuously maintain the information required to route traffic. MANET routing protocols [2] [3] are traditionally divided into three categories as shown in Figure 1, which are Proactive Routing Protocols, Reactive Routing Protocols and also Hybrid Routing Protocols. Proactive Routing Protocols [4] [5] are also called table driven routing protocols and they constantly maintain the updated topology of the network. Each node in these protocols maintains individual routing table which consists of routing information of all the nodes present in the network. Reactive Routing Protocol is also called as on-demand routing protocol. Reactive protocols do not initiate path discovery on their own, unless they are requested. Hybrid Routing Protocols [6] is a confluence of both the above protocols and contains the advantages of both of them. The routing is initially set up with a few proactively prospected routes and thereby serves the demand from additionally activated mobile nodes through reactive flooding.

\section{Related Work}

In the papers referenced up till now, a good amount of analysis is done about the performance and the effectiveness of the MANET routing protocols such as OLSR [7] [8] and DSR [9] [10]. An important aspect of the research work and studies carried out by these papers is the dealing of the QoS parameters. These parameters have been analyzed by varying the number of nodes as well as the number of effective operating nodes, changing the environment of the mobile nodes, changing the distance between them, difference in the type of application and network layer protocols chosen, etc.

Also changes in physical characteristics, data rates of transfer, RTS and fragmentation threshold, packet inter-arrival time distribution, packet size distribution are some of the other factors utilized in these studies.

In the above references of both the protocols, however only a cursory attention has been given to the performance of QoS parameters in conjunction with a

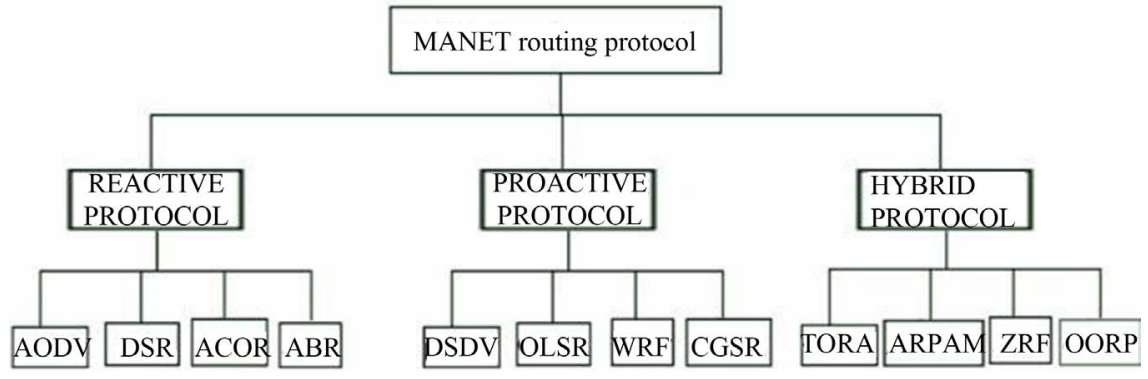

Figure 1. Categories of MANET routing protocol. 
variety of trajectories of the mobile nodes. Also performance of QoS parameters during events of mobile node failures are neither exclusively nor thoroughly dealt with in these studies for both these protocols. Hence a proper relationship in order to understand the interplay is needed. This paper attempts to establish this relation in order to develop a better understanding of the same.

\section{Research Methodology}

In order to achieve a better understanding of the QoS parameters of various trajectories, twelve simulation scenarios with 20 nodes in each of them, have been analyzed using OPNET (Optimized Network Engineering Tool) modeler version 17.5, a discrete event simulator. All nodes selected are mobile. The nodes are placed in a campus environment of $10 \mathrm{~km}$ by $10 \mathrm{~km}$ spread. The nodes can be any devices or machinery or even vehicles which are mobile and capable of wireless communication. File Transfer Protocol has been used in the study with load parameter set at high level. All nodes are assigned with a specific path trajectory which defines the mobility within the given time frame. The trajectories incorporated here are namely linear, curvilinear and mixed.

\section{Simulation Framework}

For all the above scenarios, the wireless parameters set, are shown in Table 1, as follows.

The MANET traffic generation parameters for each of the nodes are shown in Table 2 as follows.

The different results obtained have been analyzed along with their respective illustrations in the following section.

\section{Simulation and Observation}

Initially for the setup showed in Figure 2, the nodes are assigned a uniform

Table 1. Wireless parameters.

\begin{tabular}{cc}
\hline Wireless MAC Addresses & Auto Assigned \\
\hline BSS Identifier & Auto Assigned \\
Physical Characteristics & Extended Rate PHY $(802.11 \mathrm{~g})$ \\
Data Rate (bps) & $24 \mathrm{Mbps}$ \\
Channel Settings & Auto Assigned \\
Transmit Power (W) & 0.005 \\
RTS Threshold (bytes) & None \\
Fragmentation Threshold (bytes) & None \\
Short Retry Limit & 7 \\
Long Retry Limit & 4 \\
Buffer Size (bits) & 256,000 \\
Max Receive Lifetime (secs) & 0.5 \\
AP Beacon Interval (secs) & 0.02 \\
\hline
\end{tabular}


Table 2. Manet traffic parameters.

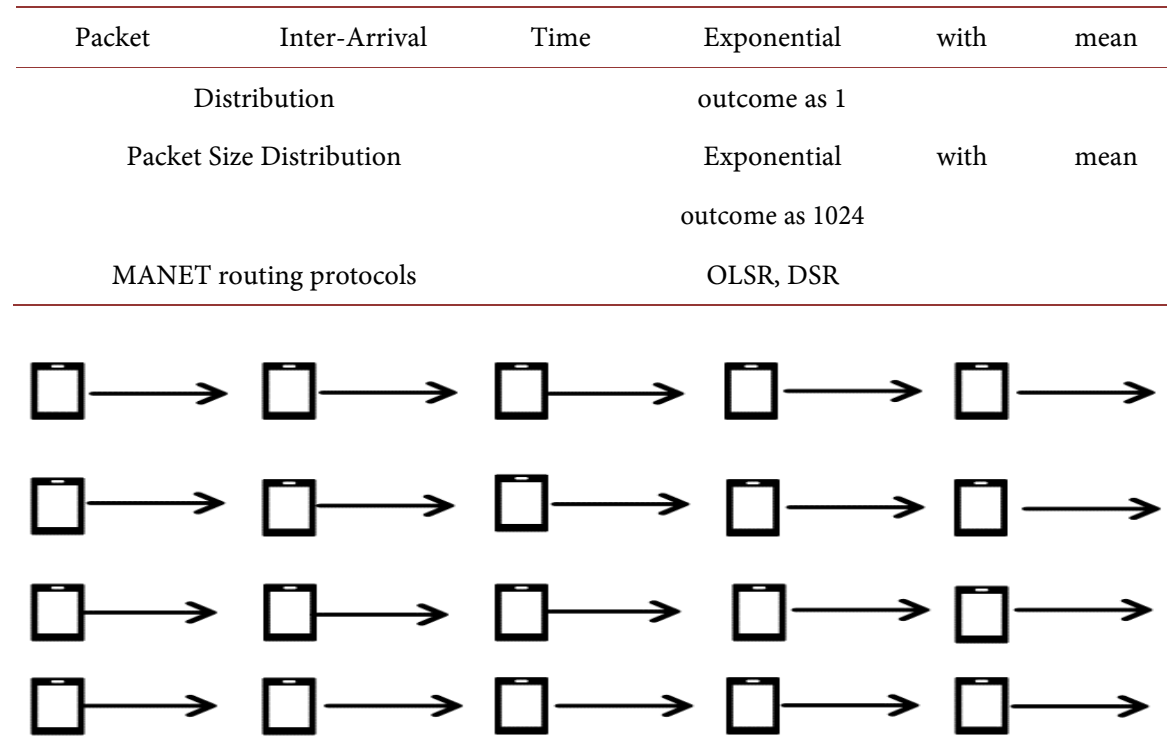

Figure 2. Setup for Scenarios 1 and 2.

linear trajectory. The protocol used is OLSR in the first scenario.

The average delay and average throughput of this scenario are shown in Figure 3 and listed in Table 3. We see that after some initial variations the curves follow a comparatively stable path. This tends to solely be a probabilistic random phenomenon.

In the second scenario the protocol used is DSR. The topology remains similar to the first scenario. The average delay and throughput of this scenario are shown in Figure 4 and listed in Table 4.

From both these figures it is observed that the average delays for both the protocols are approximately the same. But the average throughput for OLSR protocol is higher than that of DSR protocol by almost $200 \mathrm{bits} / \mathrm{sec}$. Thus OLSR gives a higher average throughput and this can be attributed to the fact that OLSR protocol is independent of the traffic and network density compared to the DSR protocol.

Up till now the cases that have been analyzed were solely belonging to the category of linear trajectory. Next we move on to curvilinear trajectory. In the setup of Figure 5 all the nodes have a curvilinear trajectory. In scenario 3, the protocol used is OLSR while in scenario 4, DSR has been used. The average delay and throughput of both these scenarios are shown in Figure 6 and Figure 7 respectively and listed in Table 5 and Table 6.

It can be inferred that the average delays for both the protocols are approximately the same. But the average throughput for OLSR protocol is higher than that of DSR protocol by almost $400 \mathrm{bits} / \mathrm{sec}$.

Now comparing the outputs for both the above setups, we see that for both the protocols, the average delay has remained almost same but the average throughputs 
Table 3. Scenario 1 Values.

\begin{tabular}{ccc}
\hline Time (in seconds) & Throughput (bits/second) & Delay (seconds) \\
\hline 0 & 487 & 0.000061 \\
0.5 & 496 & 0.000061 \\
1 & 494 & 0.000061 \\
1.5 & 494 & 0.000061 \\
2 & 495 & 0.000061 \\
2.5 & 495 & 0.000061 \\
3 & 495 & 0.000061 \\
\hline
\end{tabular}

Table 4. Scenario 2 Values.

\begin{tabular}{ccc}
\hline Time (in seconds) & Throughput (bits/second) & Delay (seconds) \\
\hline 0 & 200 & 0.000053 \\
0.5 & 299 & 0.000053 \\
1 & 300 & 0.000053 \\
1.5 & 300 & 0.000053 \\
2 & 300 & 0.000053 \\
2.5 & 300 & 0.000053 \\
3 & 300 & 0.000053 \\
\hline
\end{tabular}

Table 5. Scenario 3 Values.

\begin{tabular}{ccc}
\hline Time (in seconds) & Throughput (bits/second) & Delay (seconds) \\
\hline 0 & 2400 & 0.00011 \\
0.5 & 1000 & 0.00006 \\
1 & 1000 & 0.00006 \\
1.5 & 1000 & 0.00006 \\
2 & 1000 & 0.00006 \\
2.5 & 1000 & 0.00006 \\
3 & 1000 & 0.00006 \\
\hline
\end{tabular}

Table 6. Scenario 4 Values.

\begin{tabular}{ccc}
\hline Time (in seconds) & Throughput (bits/second) & Delay (seconds) \\
\hline 0 & 1580 & 0.00014 \\
0.5 & 600 & 0.00006 \\
1 & 600 & 0.00006 \\
1.5 & 600 & 0.00006 \\
2 & 600 & 0.00006 \\
2.5 & 600 & 0.00006 \\
3 & 600 & 0.00006 \\
\hline
\end{tabular}




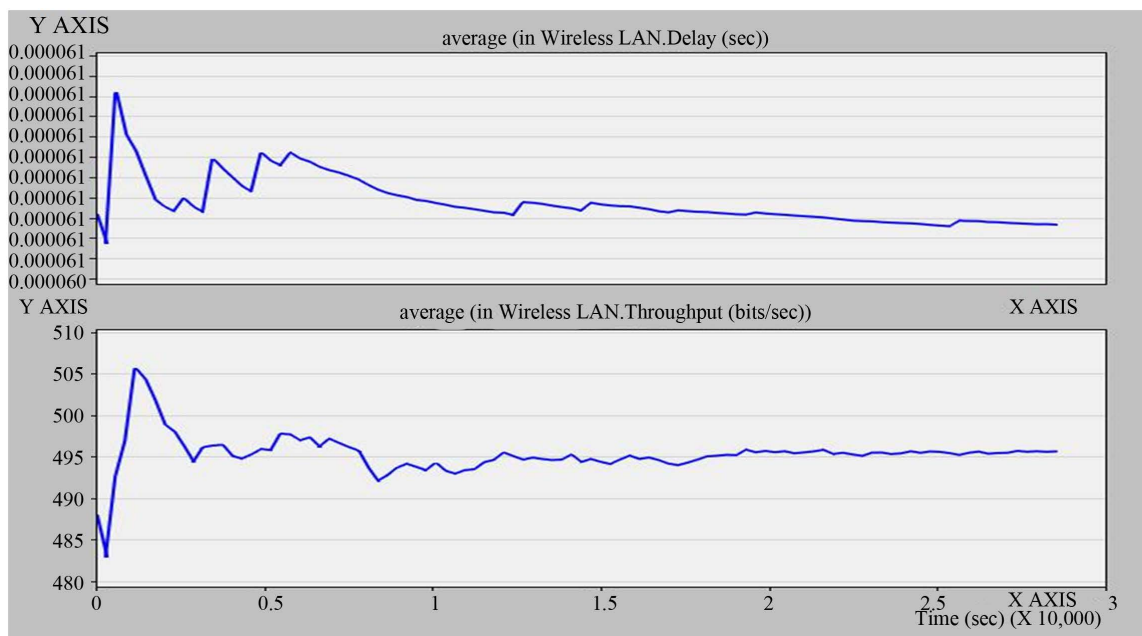

Figure 3. Output for scenario 1.

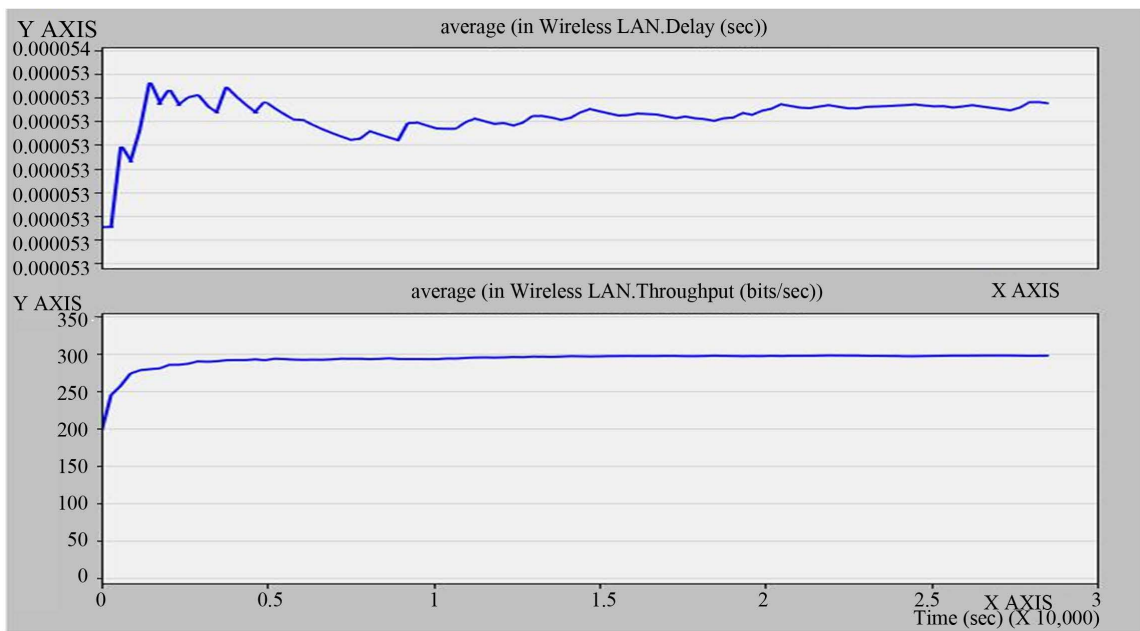

Figure 4. Output for scenario 2.

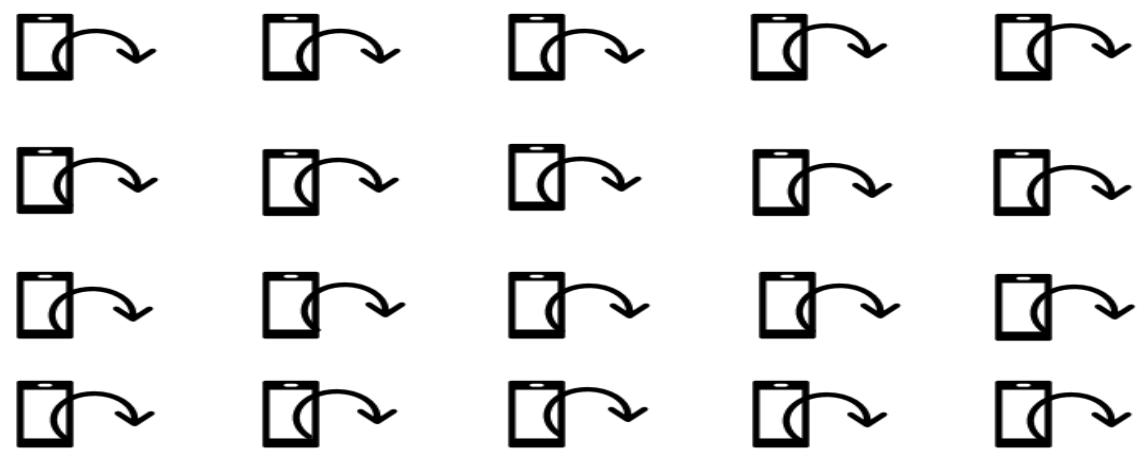

Figure 5. Setup for Scenarios 3 and 4.

have increased by a factor of almost 2 in the curved case. The average throughput for scenario 3 is about $1000 \mathrm{bits} / \mathrm{sec}$ and for scenario 4 it is approximately $600 \mathrm{bits} / \mathrm{sec}$. Thus a curved trajectory delivers a better average throughput as compared to the case of a linear trajectory of the mobile nodes. 


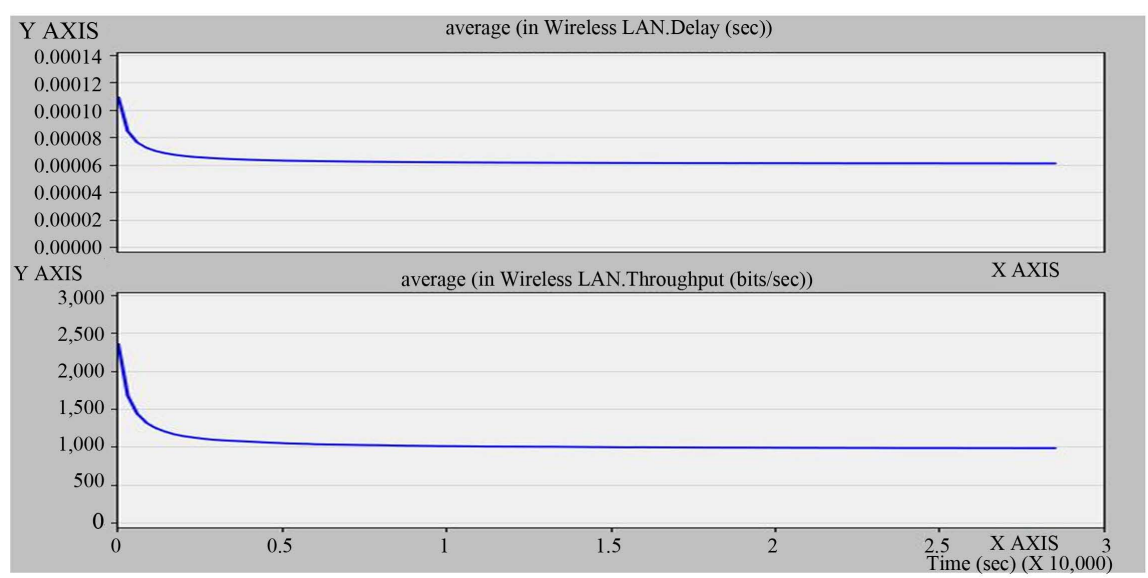

Figure 6. Output for scenario 3.

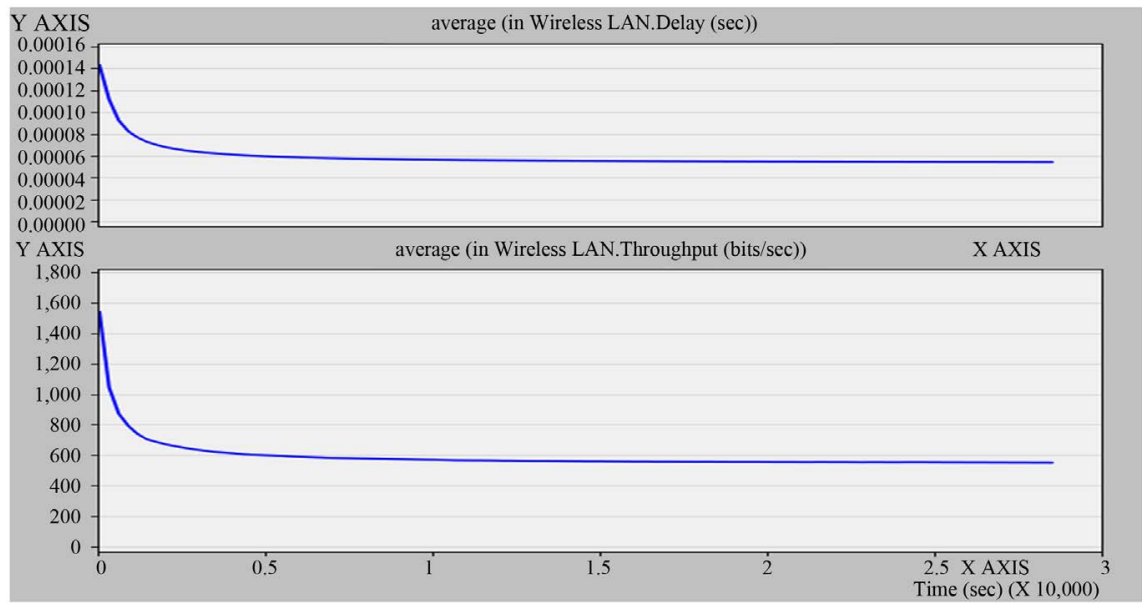

Figure 7. Output for scenario 4.

In the next setup, Figure 8, we consider linear trajectory again and this time we subject failure to few number of nodes ( 5 nodes) and do the analysis. The node selection is random.

Figure 9 and Figure 10 display the outputs for scenarios 5 and 6 respectively. They are listed down in Table 7 and Table 8 respectively.

From these outputs we see that there is no considerable effect on the average delays for both the protocols compared to the initial setup with linear trajectory and no failed nodes. But the average throughput has considerably reduced for both OLSR and DSR as compared to when there is no case of node failure.

Also here we infer that OLSR gives a higher average throughput than DSR whereas average delays remain almost unchanged. Thus effect on average throughput is more noticeable.

Now in Figure 11, we consider curvilinear trajectory again and subject failure to the same number of nodes ( 5 nodes) and continue the analysis. The node selection is random. Scenario 7 uses OLSR while scenario 8 uses DSR. Figure 12 displays the outputs for scenario 7 whereas Figure 13 displays the outputs for scenario 8. The values are listed down in Table 9 and Table 10. 


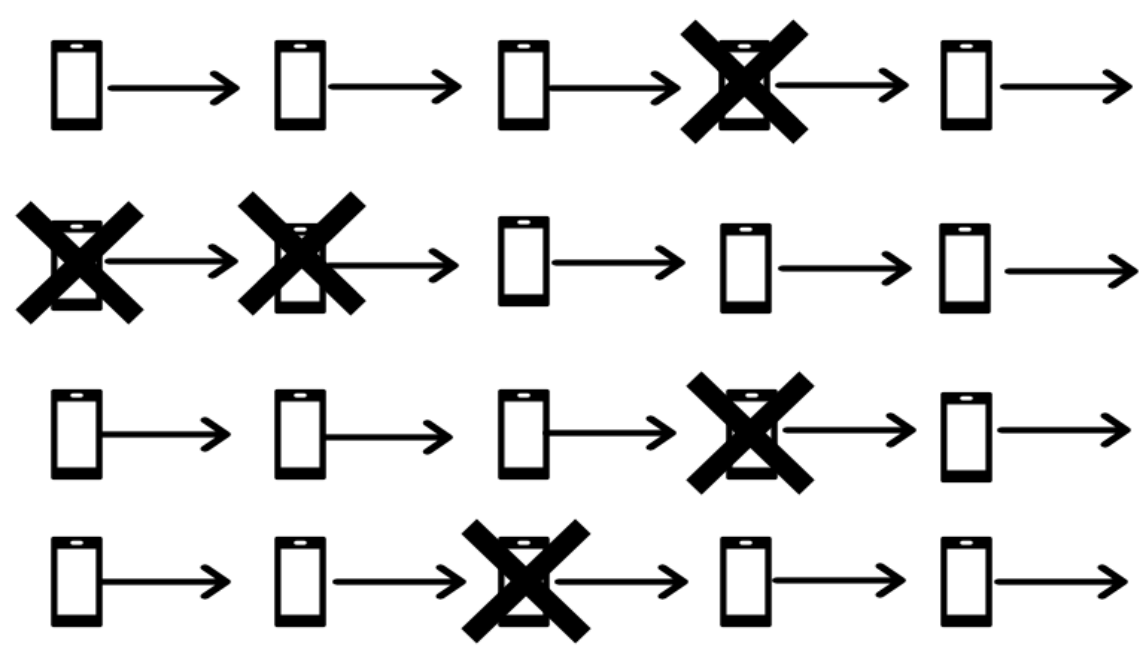

Figure 8. Setup for Scenarios 5 and 6.

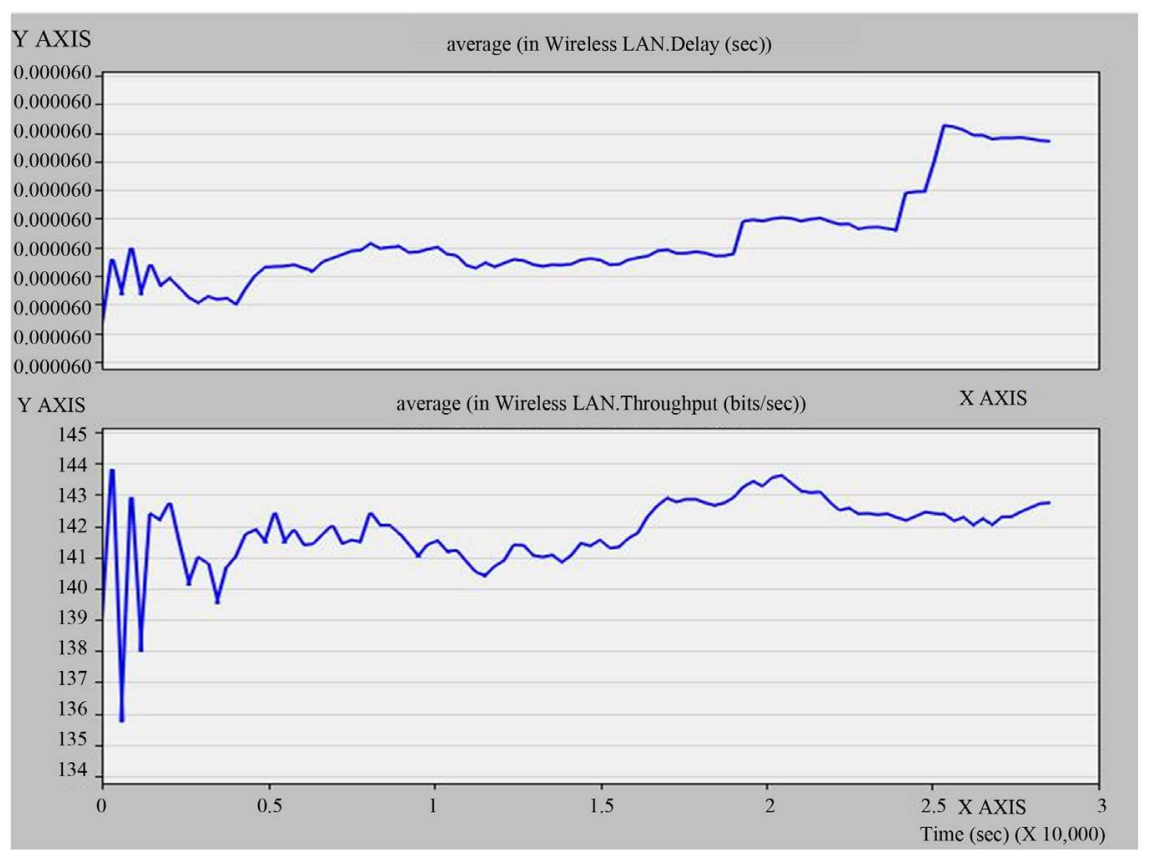

Figure 9. Output for scenario 5.

Table 7. Scenario 5 Values.

\begin{tabular}{ccc}
\hline Time (in seconds) & Throughput (bits/second) & Delay (seconds) \\
\hline 0 & 139 & 0.000060 \\
0.5 & 141.8 & 0.000060 \\
1 & 141.5 & 0.000060 \\
1.5 & 142.5 & 0.000060 \\
2 & 143.5 & 0.000060 \\
2.5 & 142.5 & 0.000060 \\
3 & 143 & 0.000060 \\
\hline
\end{tabular}




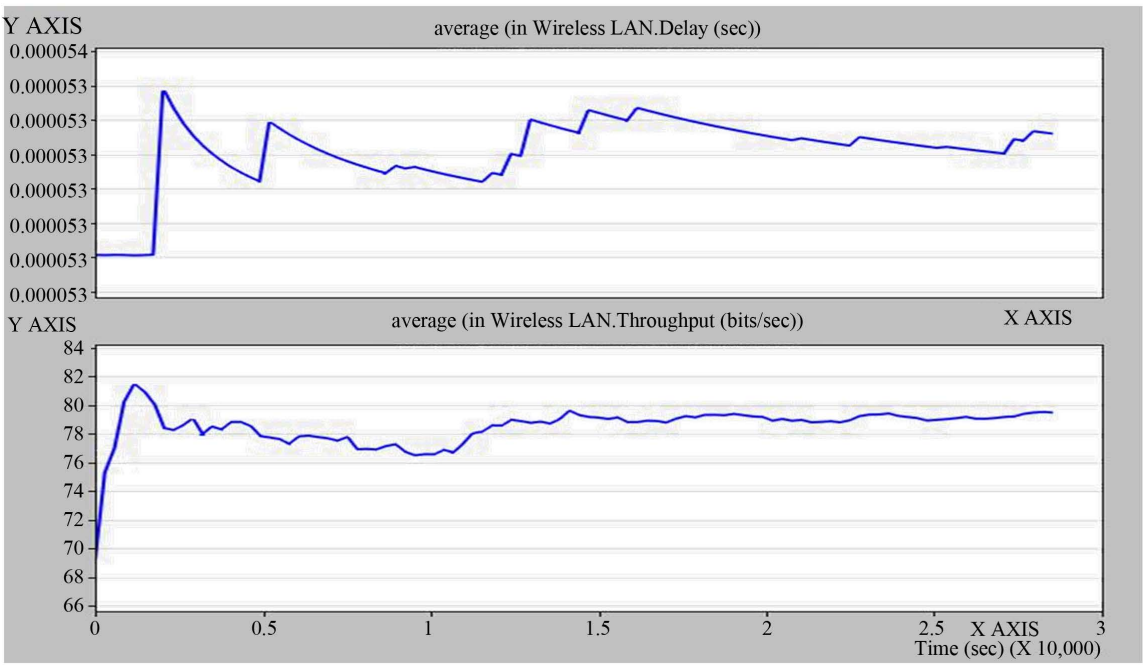

Figure 10. Output for scenario 6.

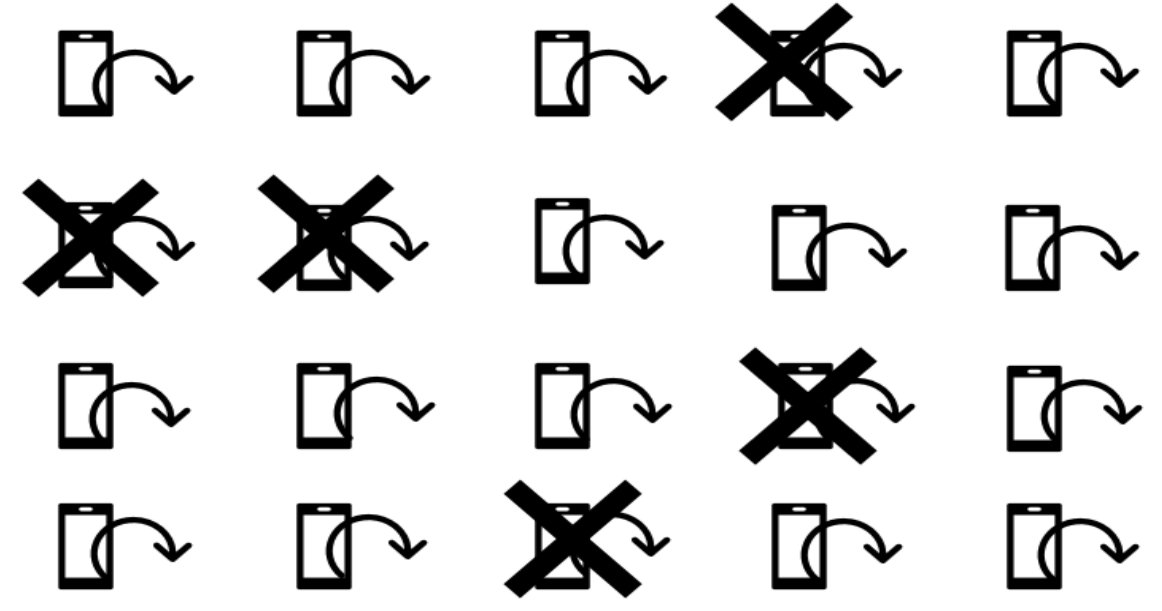

Figure 11. Setup for Scenarios 7 and 8.

Table 8. Scenario 6 Values.

\begin{tabular}{ccc}
\hline Time (in seconds) & Throughput (bits/second) & Delay (seconds) \\
\hline 0 & 70 & 0.000053 \\
0.5 & 78 & 0.000053 \\
1 & 77 & 0.000053 \\
1.5 & 79 & 0.000053 \\
2 & 79 & 0.000053 \\
2.5 & 79 & 0.000053 \\
3 & 79 & 0.000053 \\
\hline
\end{tabular}

From the outputs of Figure 12 and Figure 13 we see that there is no considerable effect on the average delays for both the protocols compared to the initial setup with curvilinear trajectory and no failed nodes. But the average 


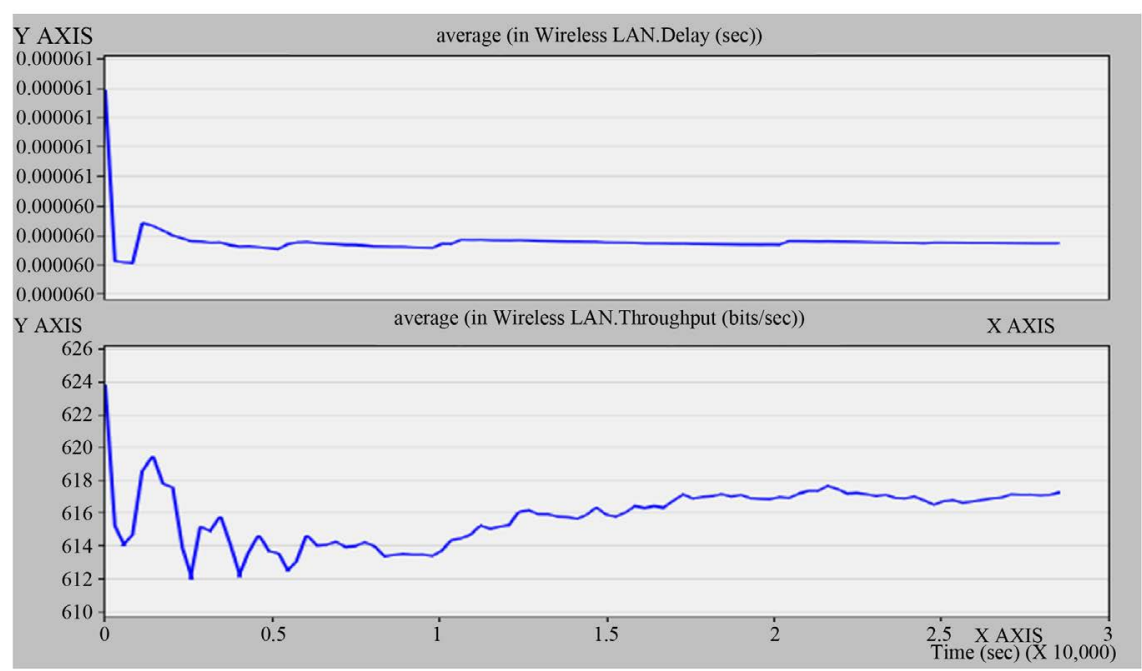

Figure 12. Output for scenario 7.

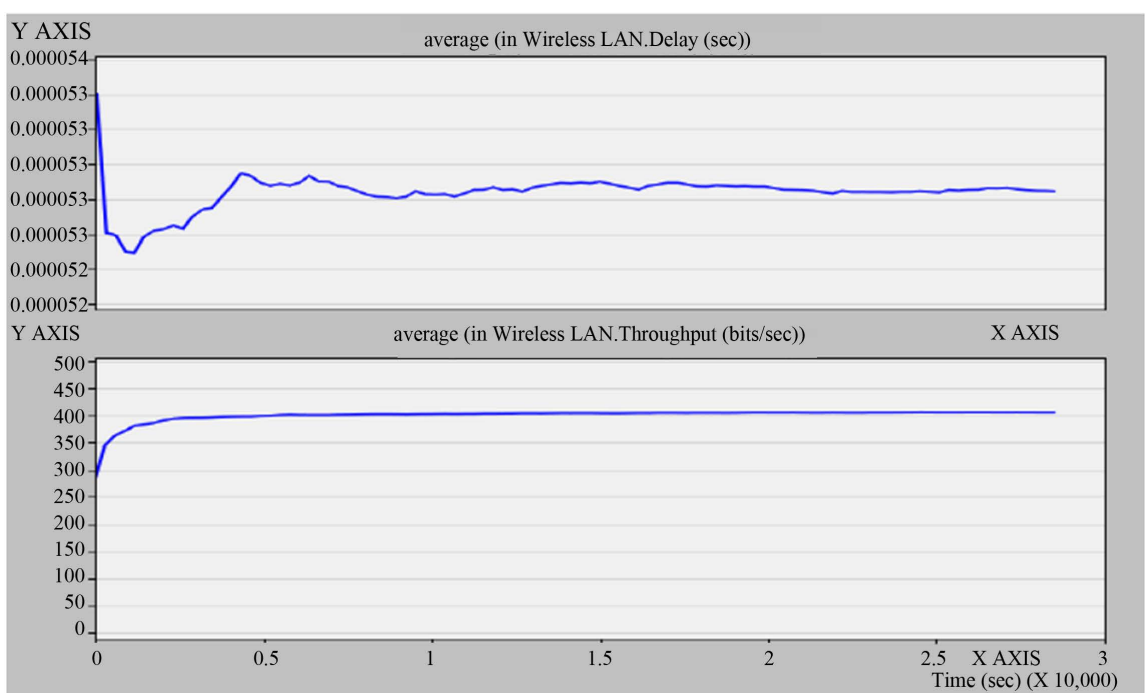

Figure 13. Output for scenario 8.

Table 9. Scenario 7 Values.

\begin{tabular}{ccc}
\hline Time (in seconds) & Throughput (bits/second) & Delay (seconds) \\
\hline 0 & 624 & 0.000061 \\
0.5 & 613.8 & 0.000060 \\
1 & 613.8 & 0.000060 \\
1.5 & 616 & 0.000060 \\
2 & 616 & 0.000060 \\
2.5 & 617 & 0.000060 \\
3 & 617 & 0.000060 \\
\hline
\end{tabular}

throughput has considerably reduced for both OLSR and DSR as compared to when there is no case of node failure. 
Table 10. Scenario 8 Values.

\begin{tabular}{ccc}
\hline Time (in seconds) & Throughput (bits/second) & Delay (seconds) \\
\hline 0 & 300 & 0.000053 \\
0.5 & 400 & 0.000053 \\
1 & 400 & 0.000053 \\
1.5 & 400 & 0.000053 \\
2 & 400 & 0.000053 \\
2.5 & 400 & 0.000053 \\
3 & 400 & 0.000053 \\
\hline
\end{tabular}

Now comparing the outputs for both these setups and the original two setups, we see that for both the protocols, the average delay has remained almost same but the average throughputs have decreased less in the curved case. Thus again, we see that, a curved trajectory delivers a better average throughput.

In the next setup, Figure 14, we consider a non-uniform, i.e., a mixed trajectory, consisting of few nodes with linear and few nodes with curved trajectories. Scenario 9 uses OLSR while scenario 10 uses DSR. Figure 15 and Figure 16 display the outputs for scenarios 9 and 10 respectively. The values are listed down in Table 11 and Table 12.

From Figure 15 and Figure 16, we see that with a certain amount of randomness added to the setup, the average delays have increased considerably for both the protocols as compared to either the linear or the curved cases. Likewise the average throughputs have also risen tremendously for both OLSR and DSR. This can be attributed to the formation of strong links between some nodes which balance the weaker ones between the others. The average delay for DSR is more as compared to OLSR while with average throughput it's the exact opposite case.

In the next setup, Figure 17, we consider mixed trajectory again and this time we subject failure to few number of nodes ( 5 nodes) and continue the analysis. The node selection is random.

Scenario 11 uses OLSR while scenario 12 uses DSR. Figure 18 and Figure 19 display the outputs for scenarios 11 and 12 respectively. The values are listed down in Table 13 and Table 14. From these outputs we see that there is no considerable effect on the average delays for both the protocols compared to the previous setup with no failed nodes.

Also OLSR gives a slightly higher average throughput than DSR in this case. For both the setups with mixed trajectory we observe that the average throughputs are almost equivalent. A possible analysis of these observations is given in the following section.

\section{Analysis}

We first observe that in the linear and curvilinear trajectories [which are essentially 


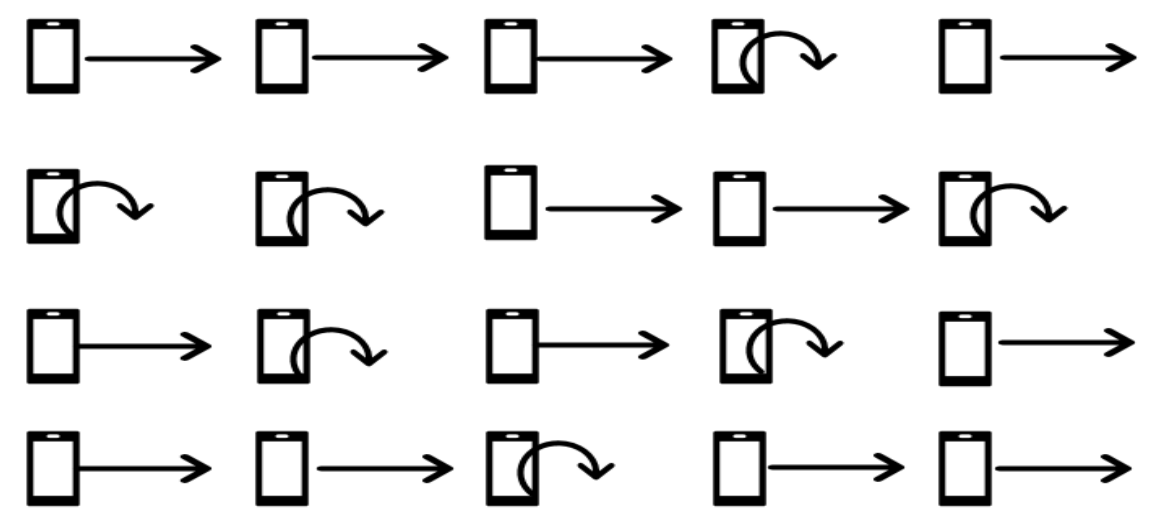

Figure 14. Setup for Scenarios 9 and 10.

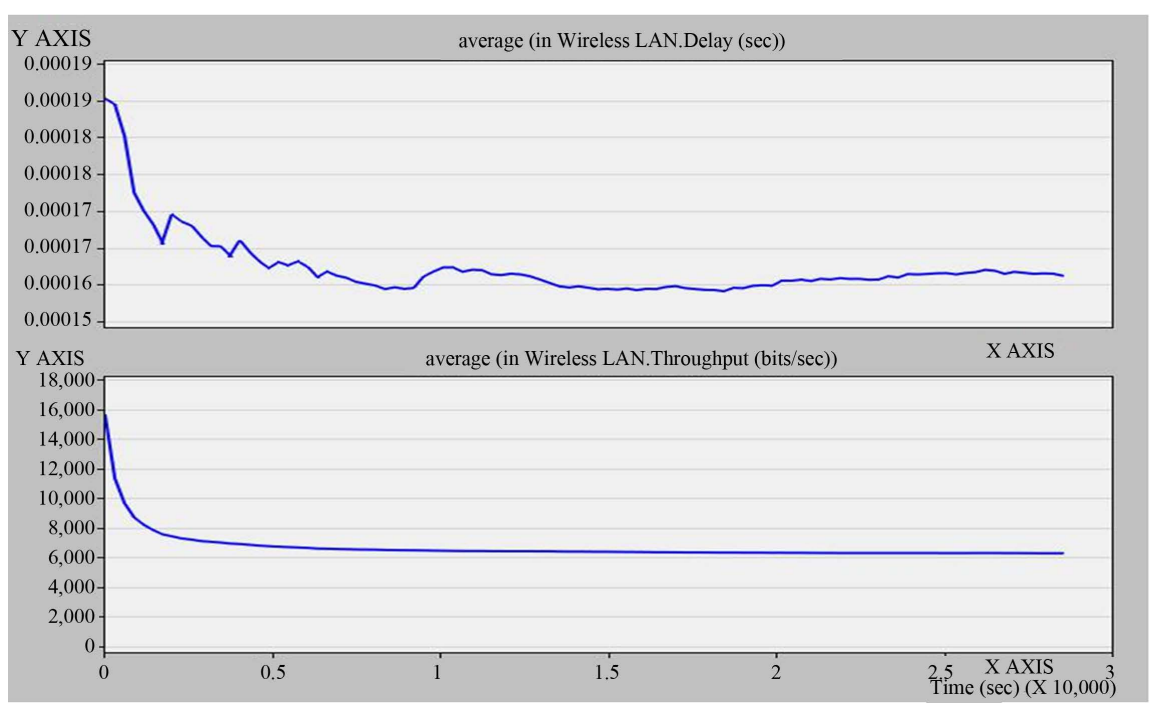

Figure 15. Output for scenario 9.

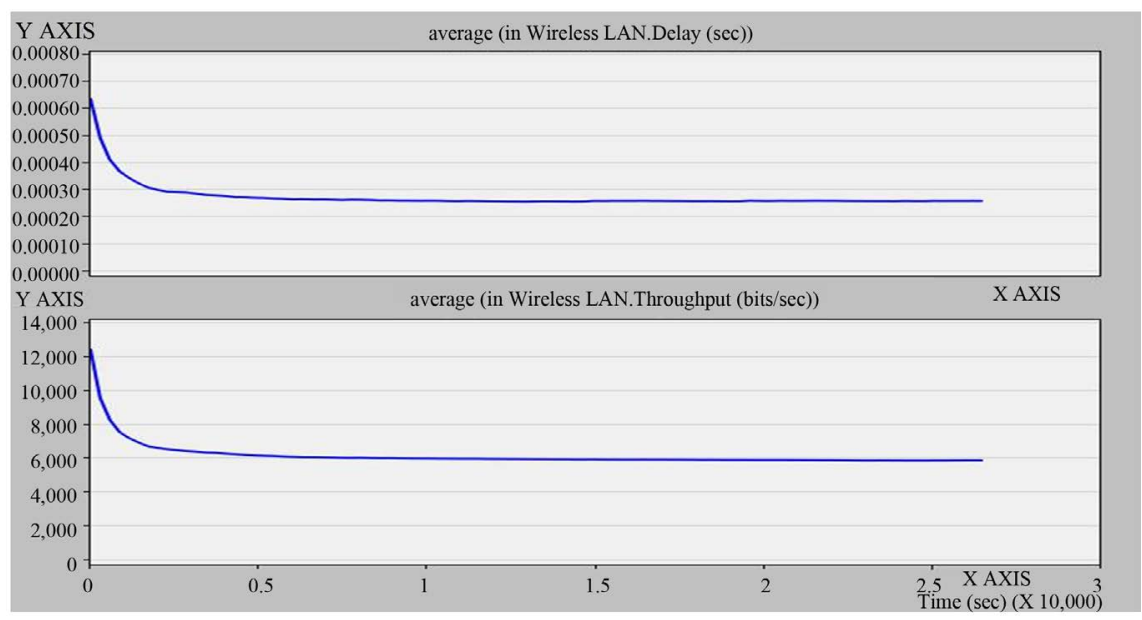

Figure 16. Output for scenario 10.

assumed constant velocity cases here], for node failures no considerable change in average delay occurs while average throughputs are reduced. Since here 


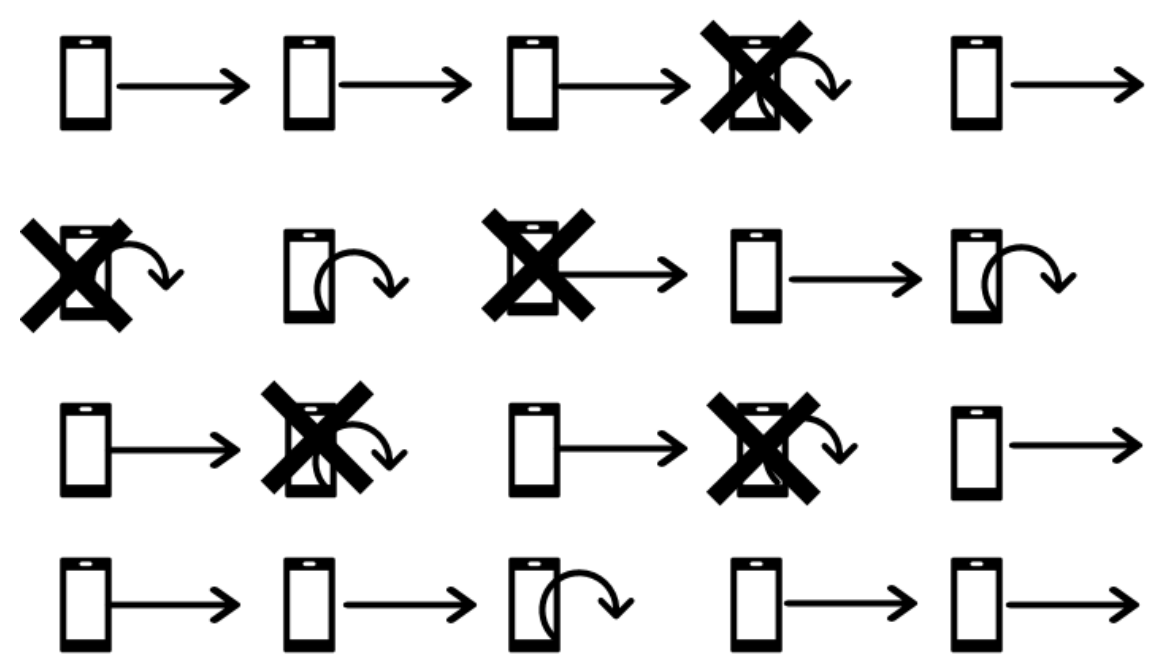

Figure 17. Setup for Scenarios 11 and 12.

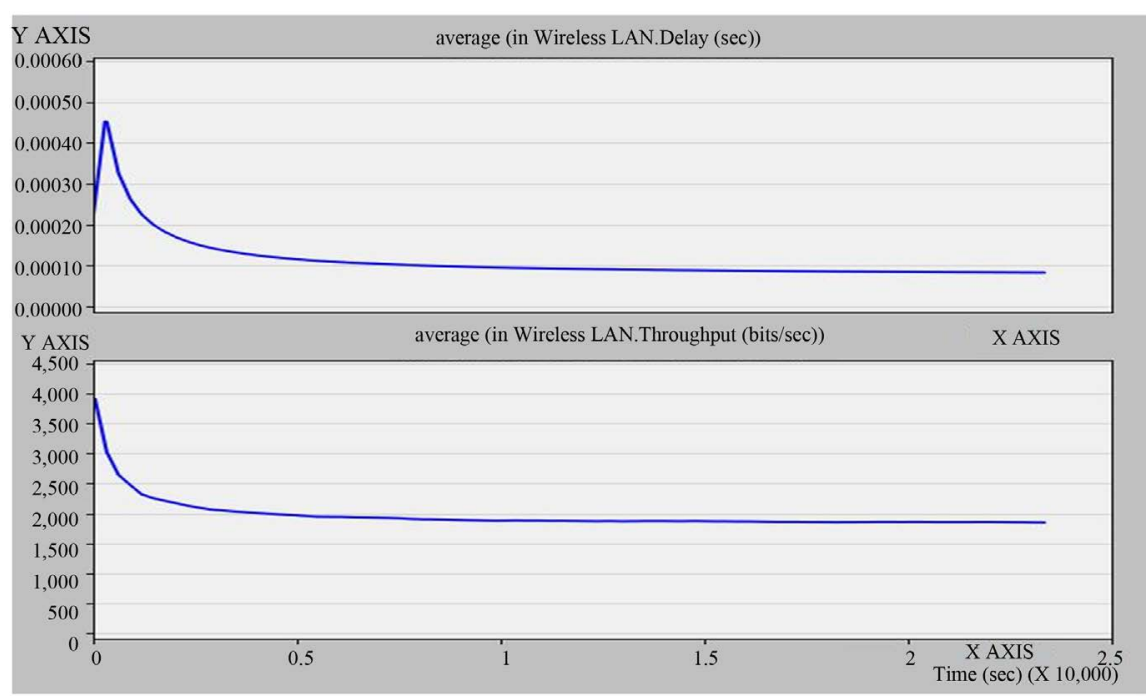

Figure 18. Output for scenario 11.

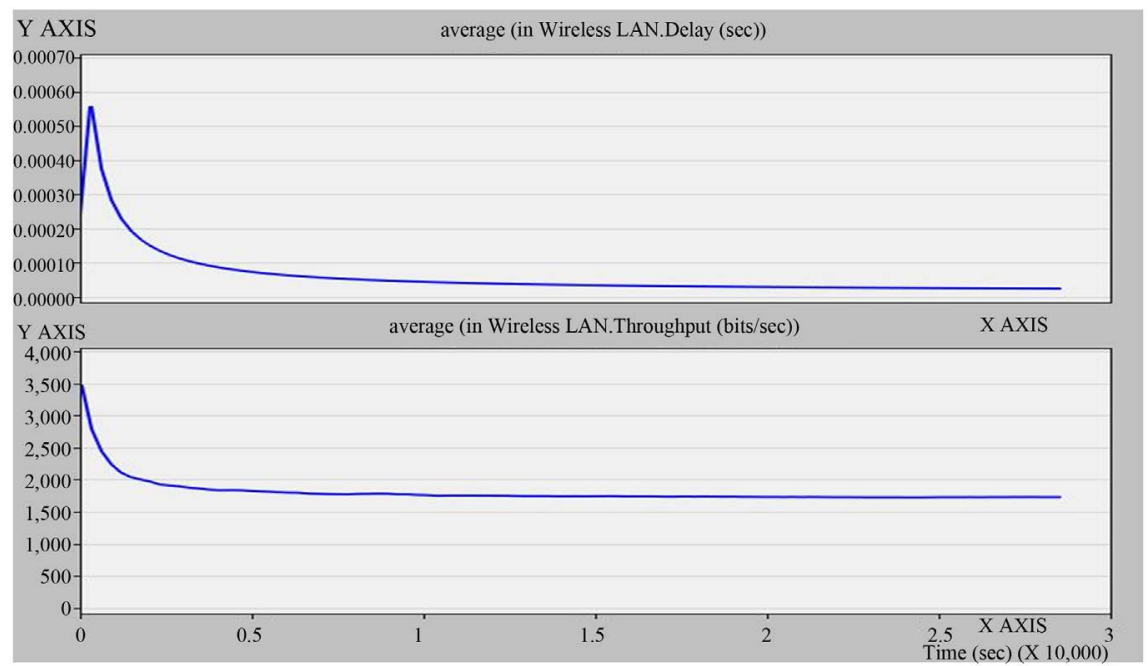

Figure 19. Output for scenario 12. 
Table 11. Scenario 9 Values.

\begin{tabular}{ccc}
\hline Time (in seconds) & Throughput (bits/second) & Delay (seconds) \\
\hline 0 & 14800 & 0.00019 \\
0.5 & 7000 & 0.000165 \\
1 & 6000 & 0.000165 \\
1.5 & 6000 & 0.00016 \\
2 & 6000 & 0.00016 \\
2.5 & 6000 & 0.000165 \\
3 & 6000 & 0.000165 \\
\hline
\end{tabular}

Table 12. Scenario 10 Values.

\begin{tabular}{ccc}
\hline Time (in seconds) & Throughput (bits/second) & Delay (seconds) \\
\hline 0 & 12200 & 0.00060 \\
0.5 & 6000 & 0.00030 \\
1 & 6000 & 0.00030 \\
1.5 & 6000 & 0.00030 \\
2 & 6000 & 0.00030 \\
2.5 & 6000 & 0.00030 \\
3 & 6000 & 0.00030 \\
\hline
\end{tabular}

Table 13. Scenario 11 Values.

\begin{tabular}{ccc}
\hline Time (in seconds) & Throughput (bits/second) & Delay (seconds) \\
\hline 0 & 3500 & 0.0025 \\
0.5 & 1800 & 0.0010 \\
1 & 1750 & 0.0008 \\
1.5 & 1750 & 0.0006 \\
2 & 1750 & 0.0004 \\
2.5 & 1750 & 0.0003 \\
3 & 1750 & 0.0003 \\
\hline
\end{tabular}

links are formed between the nodes when the routing protocol sets up; failure of nodes results in link breakages causing remaining links to be burdened with additional traffic of nodes. Thus average throughput decreases. Average delay is not much affected since route re-selection (by routing algorithm) would not bring in drastically longer paths. When we consider non-uniform trajectories, some nodes approach nearer to others while going away from the remaining ones. The former links get strengthened substantially while the latter ones get weakened. Now consider a real life scenario in Figure 20 consisting of air planes (nodes) forming an autonomous network. A node A initially sends data to another node C directly via a weak link. Now due to introduction of 
non-uniformity a strong link exists between node A and an intermediate node B and a normal one between nodes $B$ and $C$. Here, due to the availability of a powerful link between nodes $\mathrm{A}$ and $\mathrm{B}, \mathrm{A}$ would now route data to $\mathrm{B}$ and then from there to $C$. Thus, average throughout increases, as essentially the stronger links provide better interference resilient paths. But consequently, average delay increases as it is possible that many nodes now route their data through much longer paths.

In the case of node failures, throughputs are not much affected since the existence of many "super-links" and the failure of nodes mutually nullify each other. Also these links don't let the delay change much compared to the no-fail case.

\section{Conclusion}

Thus we have successfully analyzed the QoS parameters of the OLSR and DSR protocols for various scenarios and different trajectories. We infer that for the same trajectory and when number of nodes is not large, the average delay for the OLSR and DSR protocols is almost the same, whereas the latter has a lower average throughput. We also conclude that as compared to uniform trajectories like linear and curvilinear; non-uniform trajectories deliver a much better average throughput. In case of node failure, the average delays more or less remain the same whereas the average throughputs show a considerable decrease. This

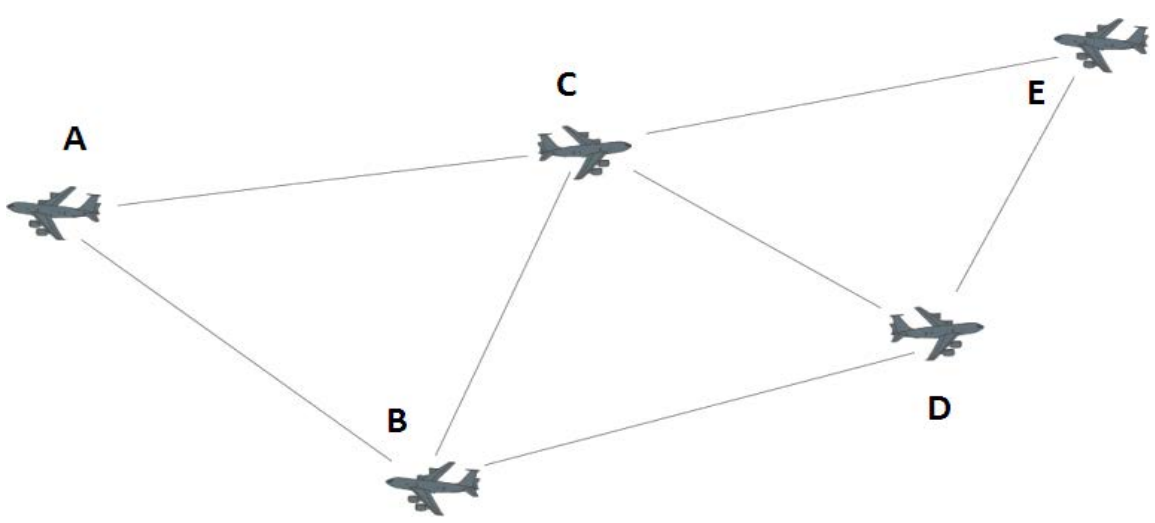

Figure 20. Example Scenario.

Table 14. Scenario 12 Values.

\begin{tabular}{ccc}
\hline Time (in seconds) & Throughput (bits/second) & Delay (seconds) \\
\hline 0 & 3500 & 0.0025 \\
0.5 & 1800 & 0.0010 \\
1 & 1750 & 0.0008 \\
1.5 & 1750 & 0.0006 \\
2 & 1750 & 0.0004 \\
2.5 & 1750 & 0.0003 \\
3 & 1750 & 0.0003
\end{tabular}


work, though extensive, has been limited due to software constraints. Effects such as that of environmental conditions could be included in this study, if not for software limitation. Also the interference caused by obstacles to communication can also be dealt with.

\section{Future Research}

The results obtained in this work can serve as the basis for similar studies involving much larger networks incorporating more number of nodes. They can also be used as reference for networks having mobile as well as stationary nodes along with various possibilities of node failures. This would serve well in determining the probabilities of failures and their possible eliminations in real life scenarios.

\section{Acknowledgements}

The authors wish to thank the staff and faculty of Sardar Patel Institute of Technology, Mumbai University and University of Arkansas at Pine Bluff for their constant support and guidance. Also the contribution of Mr. Dayanand Ambawade is much appreciated.

\section{References}

[1] Vijayalakshmi, M. and Rao, D.S. (2016) Energy Aware Multicast Clustering (EMAC) with Increased Quality of Service (QoS) in MANETs. 2 ${ }^{\text {nd }}$ International Conference on Applied and Theoretical Computing and Communication Technology, 21-23 July 2016, Bangalore, India, 793-798.

[2] Satav, P.R. and Jawandhiya, P.M. (2016) Review on Single-Path Multi-Path Routing Protocol in MANET: A Study. International Conference on Recent Advances and Innovations in Engineering (ICRAIE), 23-25 December 2016, Jaipur, India, 1-7.

[3] Liu, C. and Chang, S. (2009) The Study of Effectiveness for Ad-Hoc Wireless Network. In Proc. of ICIS $20092^{\text {nd }}$ International Conference on Interaction Sciences: Information Technology, Culture and Human, 24-26 November 2009, Seoul, Korea, 412-417. https://doi.org/10.1145/1655925.1656001

[4] Meena, R. and Tharani, L. (2015) A Review of Study of Lightweight Proactive Source Routing Protocol for MANETs. International Conference on Green Computing and Internet of Things (ICGCIoT), 8-10 October 2015, Noida, India, 1030-1034.

[5] Shenbagapriya, R. and Kumar, N. (2014) A Survey on Proactive Routing Protocols in MANETs. International Conference on Science Engineering and Management Research (ICSEMR), 27-29 November 2014, Chennai, India, 1-7.

[6] Chandra, A. and Thakur, S. (2015) Performance Evaluation of Hybrid Routing Protocols against Network Layer Attacks in MANET. $1^{\text {st }}$ International Conference on Next Generation Computing Technologies (NGCT), 4-5 September 2015, Dehradun, India, 239-244. https://doi.org/10.1109/NGCT.2015.7375119

[7] Kukreja, S. and Singh, P. (2016) Performance Metrics of AODV and OLSR in Wireless Mesh Network. $3^{\text {rd }}$ International Conference on Computing for Sustainable Global Developmnet (INDIACom), 16-18 March 2016, New Delhi, India, 3182-3185. 
[8] Mehra, R., Balli, R.S. and Kaur, P. (2016) Efficient Clustering Based OLSR Routing Protocol for VANET. Symposium on Colossal Data Analysis and Networking ( $C D A N)$, 18-19 March 2016, Indore, India, 1-7. https://doi.org/10.1109/CDAN.2016.7570915

[9] Das, S. and Singh, T. (2016) Performance Measures of Secured Routing Protocols (AODV and DSR). International Conference on Electrical, Electronics and Optimization Techniques (ICEEOT), 3-5 March2016, Chennai, India, 851-857. https://doi.org/10.1109/ICEEOT.2016.7754807

[10] Kaur, P., Kaur, D. and Mahajan, R. (2016) A Review and Comparison of AODV, DSR and ZRP Routing Protocols on the Basis of Qualitative Metrics. $3^{\text {rd }}$ International Conference on Computing for Sustainable Global Development (INDIACom), 16-18 March 2016, New Delhi, 3262-3266. 